J. Austral. Math. Soc. 22 (Series A) (1976), 501-508.

\title{
SUBGROUPS OF FINITELY PRESENTED METABELIAN GROUPS OF FINITE RANK
}

\author{
JAMES BOLER
}

(Received 6 January 1975; revised 9 April 1976)

\section{Abstract}

Let $G$ be a finitely generated metabelian group whose derived group $G^{\prime}$ has finite rank. It is shown that $G$ can be embedded in a finitely presented metabelian group $H$ with $H^{\prime}$ of finite rank.

\section{Introduction}

Baumslag (1973) has shown that each finitely generated metabelian group can be embedded in a finitely presented metabelian group. Our objective here is to prove

THEOREM 4.1. Let $G$ be a finitely generated metabelian group whose derived group $G^{\prime}$ has finite rank. Then there exists a finitely presented metabelian group $H$ with $H^{\prime}$ of finite rank, and an embedding of $G$ into $H$.

The proof of Theorem 4.1 makes use of the well-known connection between finitely generated metabelian groups and modules over finitely generated commutative rings. This relationship is mentioned in Section 1, along with some basic facts about the notion of rank. In Section 2 a residual property of certain modules is established. This property is used in Section 3 and Section 4 to prove Theorem 4.1.

\section{Preliminaries}

We denote the ring of integers by $Z$ and the field of rational numbers by $Q$.

If $x$ and $y$ are elements of a group, we use the standard notation

$$
x^{y}=y^{-1} x y ;[x, y]=x^{-1} y^{-1} x y .
$$


If $G$ is a group and $R$ is a commutative ring with unit, $R G$ is the $R$-algebra consisting of finite sums $\sum r_{i} g_{i}$ with $r_{i} \in R$ and $g_{i} \in G$. Addition is linear, and multiplication is induced by the multiplication of $G$.

If $A$ is an abelian normal subgroup of a group $G, G$ acts on $A$ by conjugation: $a g=g^{-1} a g$. Since $A$ is abelian, this gives $A$ a $(G / A)$-module structure. Equivalently, $A$ becomes a module over the integral group ring $Z(G / A)$ of $G / A$.

If $A$ is a $G$-module, the semidirect product $A] G$ of $A$ by $G$ is the group consisting of elements ag $(a \in A, g \in G)$ under the multiplication

$$
\left(a_{1} g_{1}\right)\left(a_{2} g_{2}\right)=\left(a_{1}+a_{2} g_{1}^{-1}\right)\left(g_{1} g_{2}\right) \text {. }
$$

A group $G$ is metabelian if there is an abelian normal subgroup $A$ of $G$ with $G / A$ abelian. When $G$ is finitely generated, $A$ satisfies the ascending chain condition for submodules and hence $G$ satisfies the ascending chain condition for normal subgroups (Hall, 1954).

DEFINITION 1.1. We say an abelian group $A$ has finite rank $\leqq n$ if each of its finitely generated subgroups can be generated by $n$ elements.

This notion of rank can be defined for arbitrary groups and has been called special rank (Mal'cev, 1948) and Prüfer rank (Wehrfritz, 1973).

It should perhaps be mentioned that this definition of rank is more restrictive than the usual definition of rank of an abelian group (where the rank of $A$ is the dimension of $A \otimes Q$ ). However, the two notions coincide for torsion-free abelian groups.

The following proposition is elementary.

Proposition 1.1. Let $A$ and $C$ be abelian groups of finite rank.

(i) If $B \leqq A, B$ has finite rank.

(ii) If $B$ is a homomorphic image of $A, B$ has finite rank.

(iii) If

$$
0 \rightarrow A \rightarrow B \rightarrow C \rightarrow 0
$$

is a short exact sequence of abelian groups, B has finite rank.

Proposition 1.2. Let $A$ be an abelian normal subgroup of the finitely generated group $G$, and suppose $G / A$ is abelian. If $A$ has finite rank, the torsion subgroup $T$ of $A$ is finite.

Proof. Let $T=\bigoplus_{i=1}^{x} T_{i}$ where $p_{1}, p_{2}, \cdots, p_{n}, \cdots$ are the prime numbers and $T_{i}$ consists of those elements of $T$ of order a power of $p_{i}$. Now, $T_{i}=0$ for all but finitely many primes $p_{i}$. Otherwise, putting $A_{n}=\bigoplus_{i=1}^{n} T_{i}$, 


$$
A_{1} \leqq \cdots \leqq A_{n} \leqq A_{n+1} \leqq \cdots
$$

would be an infinite chain of normal subgroups of $G$ which is proper at infinitely many places, contradicting (Hall, 1954). Thus $T=\bigoplus_{i-1}^{n} T_{i}$ for some $n$, and it is enough to show that the $p_{i}$-primary group $T_{i}$ is finite $(1 \leqq i \leqq n)$. This can be done by noticing that, again because of (Hall, 1954), there is an integer $N$ with $p_{i}^{N} t=0$ for all $t$ in $T_{i}$-otherwise a properly ascending infinite chain

$$
A_{1} \leqq \cdots \leqq A_{j} \leqq A_{i+1} \leqq \cdots
$$

could be constructed by letting

$$
A_{i}=\left\{t \in T_{i} \mid P_{i}^{i} t=0\right\} .
$$

Thus $T_{i}$ is a $p_{i}$-primary abelian group with a finite exponent hence is a direct sum of cyclic groups (see Kurosh (1955), p. 171). Now $T_{i} \leqq A$ has finite rank by Proposition 1.1, and since $T_{i}$ has finite exponent, it must be finitely generated. This can be seen by noting that a direct sum of $m$ cyclic groups of order $p^{n}, p$ a prime and $n$ fixed, cannot be generated by fewer than $m$ elements. Thus $T_{i}$ is finite and Proposition 1.2 is proved.

\section{Separating subspaces of $Q T$-modules}

Throughout this section $T$ is a finitely generated abelian group. In order to prove Theorem 4.1 , we will make use of

THEOREM 2.1. Let $A$ be a finitely generated $Q T$-module and $0 \neq S$ a subspace of $A$ with $\operatorname{dim}_{Q} S<\infty$. Then there is a submodule $K$ of $A$ with $\operatorname{dim}_{Q}(A / K)<\infty$ and $K \cap S=0$.

Note that Theorem 2.1 implies that nonintersecting finite-dimensional subspaces of $A$ can be separated by a submodule of finite codimension.

Proof of TheOREM 2.1. We shall prove Theorem 2.1 by a sequence of lemmas. Following Hall (1959), we say a $Q T$-module $A$ is monolithic with monolith $M$ if the intersection $M$ of the nonzero submodules of $A$ is itself nonzero.

LEMMA 2.1. Let $R$ be a noetherian algebra over a field $F$ and let $I$ be an ideal of $R$ with $I^{m}=0$ for some positive integer $m$. If $\operatorname{dim}_{F}(R / I)<\infty$, then $\operatorname{dim}_{F} R<\infty$.

Proof. The lemma is clearly true when $m=1$. Let $m \geqq 2$ and suppose it is true for $m-1$. Then there is a short exact sequence

$$
0 \rightarrow I^{m-1} \rightarrow R \rightarrow R / I^{m-1} \rightarrow 0
$$


of $R$-modules. Since $\left(I / I^{m-1}\right)^{m-1}=I^{m-1}, \operatorname{dim}_{F}\left(R / I^{m-1}\right)<\infty$ by induction. Now $I^{m-1}$ is finitely generated because $R$ is noetherian, and $I\left(I^{m-1}\right)=0$. Thus $I^{m-1}$ is a finitely generated $R / I$-module, so that the finite dimensionality of $R / I$ implies that of $I^{m-1}$. Thus

$$
\operatorname{dim}_{F} R=\operatorname{dim}_{F} I^{m-1}+\operatorname{dim}_{F}\left(R / I^{m-1}\right)<\infty
$$

and Lemma 2.1 is proved.

LeMma 2.2. Let $R$ be a homomorphic image of $Q T$ and suppose $R$ is monolithic with monolith $M$. Then each element of $I=\{r \in R \mid r M=0\}$ is nilpotent.

Proof. Let $u \in I$ and suppose $u$ is not nilpotent. Then the elements

$$
u, u^{2}, \cdots, u^{n}, \cdots
$$

are all nonzero and hence each $R u^{n}(n=1,2, \cdots)$ is a nonzero ideal of $R$ and so must contain $M$. If $0 \neq a \in M$ is fixed, we must have

$$
y_{1}, y_{2}, \cdots, y_{n}, \cdots
$$

in $R$ with $y_{n} u^{n}=a$.

Now let $A_{n}=\left\{r \in R \mid r u^{n}=0\right\}$ so that

$$
A_{1} \subseteq A_{2} \subseteq \cdots \subseteq A_{n} \subseteq A_{n+1} \subseteq \cdots
$$

is an infinite ascending sequence of submodules of $R$. Now $y_{n} u^{n+1}=a u=0$ since $u \in I$. Thus $y_{n} \in A_{n+1}-A_{n}$ and hence (2.1) is a properly ascending infinite chain, a contradiction since each finitely generated $Q T$-module satisfies the ascending chain condition for submodules (Hall, 1959, Theorem 4). Thus Lemma 2.2 is proved.

Lemma 2.3. Let $R$ be a homomorphic image of $Q T$. If $R$ is monolithic, $\operatorname{dim}_{Q} R<\infty$.

Proof. Let $M$ be the monolith of $R$ and let $I=\{r \in R \mid r M=0\}$. By Lemma 2.2 each element of $I$ is nilpotent. Since $I$ is finitely generated, there is an integer $m$ with $I^{m}=0$.

Now $M$, being the monolith of $R$, is irreducible, so that $M \approx R / I$ and $I$ is a maximal ideal of $R$. By the Hilbert Nullstellensatz (see Atiyah-MacDonald (1969), p. 67), $\operatorname{dim}_{Q}(R / I)<\infty$. Invoking Lemma 2.1 completes the proof of Lemma 2.3 .

Lemma 2.4. Let $A$ be a finitely generated monolithic $Q T$-module. Then $\operatorname{dim}_{Q} A<\infty$. 
Proof. Let $M$ be the monolith of $A$ and let $a_{1}, \cdots, a_{k}$ be a set of nonzero generators for $A$. If $R=Q T$, then each $R a_{i}(1 \leqq i \leqq k)$ is a nonzero submodule of $A$ and so must contain $M$. It follows that each $R a_{i}$ is monolithic with monolith $M$. By Lemma 2.3,

$$
\operatorname{dim}_{Q} R a_{i}<\infty \quad(1 \leqq i \leqq k) .
$$

But $A$ is a homomorphic image of $\bigoplus_{i=1}^{k} R a_{i}$, so that

$$
\operatorname{dim}_{Q} A \leqq \sum_{i=1}^{k} \operatorname{dim}_{Q}\left(R a_{i}\right)<\infty .
$$

LEMMA 2.5. Let $A$ be a finitely generated $Q T$-module and let $0 \neq a \in A$. Then there is a submodule $K$ of $A$ with $a \notin K$ and $\operatorname{dim}_{O}(A / K)<\infty$.

Proof. Let $K$ be a submodule of $A$ maximal with respect to $a \notin K$. Then each nonzero submodule of $A / K$ contains $a+k$, so that $A / K$ is monolithic. By Lemma 2.4, $\operatorname{dim}_{Q}(A / K)<\infty$ and Lemma 2.5 is proved.

Proof of THEOREM 2.1. Let $A$ be a finitely generated $Q T$-module and $S$ a nonzero subspace of $A$ with basis $x_{1}, \cdots, x_{n}$. We argue by induction on $n$.

If $n=1$, the result follows from Lemma 2.5 .

Assume $n>1$ and that the theorem holds for subspaces of dimension less than $n$. Let

$$
S_{0}=Q x_{1}+\cdots+Q x_{n-1} .
$$

Then there is a submodule $K_{0}$ with $K_{0} \cap S_{0}=0$ and $\operatorname{dim}_{Q}\left(A / K_{0}\right)<\infty$. If $S \cap K_{0}=0$, we're done. If not, let

$$
0 \neq y=a_{1} x_{1}+\cdots+a_{n} x_{n} \in S \cap K_{0} \quad\left(a_{1}, \cdots, a_{n} \in Q\right) .
$$

Then $a_{n} \neq 0$, so that $z=y a_{n}^{-1}$ lies in $S \cap K_{0}$ and

$$
x_{1}, \cdots, x_{n-1}, z
$$

is a basis for $S$. It follows that $S \cap K_{0}=Q z$.

Invoking Lemma 2.5 again, let $K_{1}$ be a submodule with $z \notin K_{1}$ and $\operatorname{dim}_{Q}\left(A / K_{1}\right)<\infty$. Putting $K=K_{0} \cap K_{1}$, we have

$$
\operatorname{dim}_{Q}(A / K) \leqq \operatorname{dim}_{Q}\left(A / K_{0}\right)+\operatorname{dim}_{Q}\left(A / K_{1}\right)<\infty
$$

and

$$
S \cap K=S \cap\left(K_{0} \cap K_{1}\right)=Q z \cap K_{1}=0 .
$$

This completes the proof of Theorem 2.1. 


\section{Embedding torsion-free groups}

In this section we begin the process of deducing Theorem 4.1 from Theorem 2.1. At this point it is convenient to introduce the theorem of Baumslag (1973) mentioned in the introduction. We state this result in the following form.

THEOREM 3.1 (Baumslag, 1973). Let $A$ be an abelian normal subgroup of a finitely generated group $G$ with $G / A$ abelian. Then there is a finitely presented semidirect product $H=B] U$ with $B$ and $U$ abelian, and an embedding $\phi$ of $G$ into $H$. $\phi$ may be chosen so that $\phi(A) \subseteq B$.

We now use Theorems 2.1 and 3.1 to deduce

THEOREM 3.2. Let $G$ be a finitely generated metabelian group. Suppose $G$ has a normal subgroup $A$ which is maximal with repect to $A$ and $G / A$ both being abelian, and which is torsion free and of finite rank. Then there is a finitely presented group $L=C] U$, with both $C$ and $U$ abelian and of finite rank, and an embedding $\psi$ of $G$ into $L$. We may choose $L$ so that $C$ is torsion-free and $\psi(A) \subseteq C$.

Proof. Let $A$ and $G$ be as in the statement of the theorem and let $\phi$ : $G \rightarrow B] U=U H$ be given by Theorem 3.1. In particular, $\left.\phi\right|_{A}: A \rightarrow B$ is an embedding. Tensoring with the field of rational numbers, we obtain maps

$$
A \stackrel{i}{\rightarrow} A \otimes Q \stackrel{\phi_{A} \otimes 1}{\longrightarrow} B \otimes Q
$$

where $i(a)=a \otimes 1$. Because $A$ is torsion-free and $Q$ is a flat $Z$-module, the mapping $\beta=\left(\left.\phi\right|_{A} \otimes 1\right) \circ i$ is an embedding of $A$ into $B \otimes Q$.

Let $\gamma: B] U \rightarrow(B \otimes Q)] U$ be defined by

$$
\gamma(b u)=(b \otimes 1) u \quad(b \in B, u \in U)
$$

Now $\beta(A) \subseteq A \otimes Q$ and since $A$ has finite rank, $A \otimes Q$ is a finite dimensional subspace of $B \otimes Q$. By Theorem 2.1, there is a submodule $K$ of $B \otimes Q$ with

$$
\operatorname{dim}_{o}((B \otimes Q) / K)<x \text { and } K \cap \beta(A)=0 .
$$

Let

$$
\left.\delta:(B \otimes Q)] U \rightarrow\left(\frac{B \otimes Q}{K}\right)\right] U
$$

be defined by

$$
\delta((b \otimes q) u)=((b \otimes q)+K) u
$$


Then $\delta \gamma \phi$ is a map of $G$ into $\delta \gamma H$. By the constructions above, $\delta \gamma H=D] U$, where $D=\delta \gamma B$ and $\delta \gamma \phi A \subseteq D$. Now, $D$ has finite rank since it is a subgroup of the finite rank group $\left(\frac{B \otimes Q}{K}\right)$. Since $A$ is a maximal abelian subgroup of $G, \phi(G) \cap B=\phi(A)$ and $\delta \gamma \phi G \cap D=\delta \gamma \phi A$. It follows that $\delta \gamma \phi$ is an embedding of $G$ into $D] U$, with $D$ having finite rank.

Now since $D$ has finite rank, it torsion part $F$ is finite by Proposition 1.2. Since $A$ is torsion-free, $\delta \gamma \phi A \cap F=0$. Thus $\delta \gamma \phi G$ embeds in $\left.\left(\frac{D}{F}\right)\right] U$. Let

$$
\left.\eta: D] U \rightarrow \frac{D}{F}\right] U \quad \text { be defined by } \quad \eta(d u)=(d+F) u .
$$

Now putting

$$
C=D / F \quad L=C] U \quad \psi=\eta \delta \gamma \phi
$$

we see that the conclusion of Theorem 3.2 is satisfied: the fact that $L$ is finitely presented follows from its being a factor group of the finitely presented metabelian group $H$. Because of the maximum condition for normal subgroups, any factor group of a finitely presented metabelian group is finitely presented. This completes the proof of Theorem 3.2.

\section{Conclusion of proof of Theorem 4.1}

Let $G$ be a finitely generated metabelian group whose derived group $G^{\prime}$ has finite rank. Let $G / G^{\prime}=T$ and let $F$ be the torsion subgroup of $G^{\prime}$, which is finite by Proposition 1.2. Let $G_{0}=G / F$ and let $A_{1} / F$ be a normal subgroup of $G_{0}$ containing $G^{\prime} / F$ and maximal with respect to being abelian. Let $F_{1} / F$ be the torsion subgroup of $A_{1} / F$. Now let $G_{1}=G / F_{1}$ and iterate this process to obtain sequences

$$
\begin{gathered}
F=F_{0} \subseteq F_{1} \subseteq \cdots \subseteq F_{i} \subseteq F_{i+1} \subseteq \cdots \\
A^{\prime}=A_{11} \subseteq A_{1} \subseteq \cdots \subseteq A_{1} \subseteq A_{i+1} \subseteq \cdots
\end{gathered}
$$

of normal subgroups of $G$ with $F_{i+1} / F_{i}$ finite and $A_{i} / F_{i-1}$ maximal in $G_{i-1}=G / F_{i-1}$ with respect to being abelian $(i=1,2, \cdots)$. Since $G$ satisfies the ascending chain condition for normal subgroups, there is an integer $n$ with $F_{n}=F_{n+1}$ and $A_{n}=A_{n+1}$. Then $G_{n}$ has a torsion-free normal subgroup $A_{n} / F_{n}$ which is maximal with respect to being abelian. Since $G^{\prime} \subseteq A_{n}, A_{n} / F_{n}$ has finite rank by Proposition 1.1. By Theorem 3.2, there is an embedding $\psi$ of $G_{n}$ into a finitely presented metabelian group $L=C] U$ with $C$ of finite rank. Since $L^{\prime} \subseteq C, L^{\prime}$ also has finite rank. 
that finitely generated metabelian groups are residually finite, there is a normal subgroup $N$ of $G$ with $N \cap F_{n}=1$ and $G / N$ finite. The map $\zeta$ : $G \rightarrow L \times G / N$ defined by $\zeta(g)=\left(\psi\left(g F_{n}\right), g N\right)$ has kernel $N \cap F_{n}=1$ and so is an embedding into $L \times G / N$, which is clearly finitely presented and metabelian. Since $L^{\prime}$ has finite rank and $G / N$ is finite, $(L \times G / N)^{\prime}=$ $L^{\prime} \times(G / N)^{\prime}$ clearly has finite rank, and the proof of Theorem 4.1 is complete.

\section{Acknowledgements}

This article is based on a chapter of the author's thesis (Boler, 1973). I should like to take this opportunity to thank my supervisor Gilbert Baumslag for his advice and encouragement. 1 am also grateful to Karl Gruenberg for his help.

The seminal work of Hall $(1954,1959,1961)$ on finitely generated soluble groups has been used throughout this work. In particular, some of the arguments of the proof of Theorem 2.1 are based on similar arguments of (Hall, 1959).

\section{References}

M. F. Atiyah and I. G. MacDonald (1969), Introduction to Commutative Algebra (AddisonWesley, Reading, Massachusetts; London; Don Mills, Ontario).

Gilbert Baumslag (1973), 'Subgroups of finitely generated metabelian groups', J. Austral. Math. Soc. 16, 98-110.

James S. Boler (1974), 'Embedding and conjugacy in metabelian groups', (PhD thesis, Rice University, Texas).

P. Hall (1954), 'Finiteness conditions for soluble groups', Proc. London Math. Soc. (3) 4, 419-436.

P. Hall (1959), 'On the finiteness of certain soluble groups', Proc. London Math. Soc. (3) 9, $595-622$.

P. Hall (1961), 'The Frattini subgroups of finitely generated groups', Proc. London Math. Soc. (3) 11, 327-352.

A. G. Kurosh (1955), Theory of Groups I (translated by K. A. Hirsch) (Chelsea, New York, New York).

А. И. Малыцев [А. І. Mal'cev], 'O групгах коречиого ранга' [On groups of finite rankl, Math. Sh. N.S. 22 (64), 351-352.

B. A. F. Wehrfritz (1973), Infinite Linear Groups. An account of the Group-Theoretic Properties of Infinite Groups of Matrices (Ergebnisse der Mathematik und ihrer Grenzgebiete, 76. Springer-Verlag, Berlin, Heidelberg, New York).

Department of Mathematics,

Oklahoma State University,

Stillwater, Oklahoma 74074, U.S.A. 